\title{
Educación democrática en el Paraguay Una mirada diferente
}

\section{Lidia Valentina Cuevas}

lilavalent@gmail.com

Cátedra Historia Diplomática del Paraguay y Derecho Electoral

Facultad de Derecho, Ciencias Políticas y Sociales Cátedra Comunicación Oral y Escrita y Metodología de la Investigación I Facultad de Ciencias Contables, Administrativas y Económicas

Dorado (pez) c/ Tajy Sa'yju (lapacho amarillo) 1000 viviendas, manzana 15, lote 5. Ayolas. Misiones. Paraguay

\section{RESUMEN}

Se pretende, conceptualizar, la función educativa paraguaya, en relación al contexto de la democracia, y evaluar la adquisición de competencias democráticas, vinculadas a experiencias de Centros de Estudiantes de colegios del nivel medio, casos UNEPY., y FENAES., se utilizó el método cualitativo descriptivo, análisis desde la interpretación bibliográfica, entrevistas dirigidas y encuestas, se reflexiona sobre la Educación a partir del discurso del Presidente Rodríguez, Giménez y Ríos (2015), sostienen: "se adhería a la ecuación epistémica democrática: la exigencia para un país que pretende ser democrático, es igual a la implementación de la economía de mercado”..., se conceptualiza el problema de la Política Educativa, está en la esencia de su enfoque, falta sincronización entre el Estado social de derecho, y el correlativo ideológico de una democracia neoliberal, Velázquez Seiferheld (2018), puntualizó: ciertas características consideradas como variables se enmarcan en el capitalismo, coincidiendo, con (Estébanez, 1997), quien en su obra “Filosofía de la Educación”, sostiene la mesología, traducida en la práctica del pragmatismo exacerbado, sin generar aprendizajes significativos. Resultados de encuestas y entrevistas destacan la transversalidad del componente Educación democrática en el currículum de la educación media como debilidad.

Palabras claves: educación; democracia y competencias democráticas 


\title{
Democratic education in Paraguay
}

\section{A different look}

\begin{abstract}
It is intended to conceptualize the Paraguayan educational function, in relation to the context of democracy, and to evaluate the acquisition of democratic competences, linked to experiences of Student Centers of middle-level schools, cases UNEPY., And FENAES., The descriptive qualitative method, analysis from the bibliographic interpretation, directed interviews and surveys, it is reflected on Education from the speech of President Rodríguez, Giménez and Ríos (2015), maintain: "it adhered to the democratic epistemic equation: the requirement for a country that pretends to be democratic, is equal to the implementation of the market economy "..., the problem of Educational Policy is conceptualized, it is in the essence of its approach, there is a lack of synchronization between the social rule of law, and the ideological correlation of a neoliberal democracy, Velázquez Seiferheld (2018), pointed out: certain characteristics considered as variables are framed in capitalism, coinciding, with (Estébanez, 1997), who in his work "Philosophy of Education", supports mesology, translated into the practice of exacerbated pragmatism, without generating significant learning. Results of surveys and interviews highlight the transversality of the Democratic Education component in the high school curriculum as a weakness.
\end{abstract}

Keywords: education; democracy and democratic powers

Artículo recibido: 02 Setiembre. 2021 Aceptado para publicación: 30 Setiembre. 2021

Correspondencia: lilavalent@gmail.com Conflictos de Interés: Ninguna que declarar 


\section{INTRODUCCIÓN}

El proceso educativo, explica Filmus (2005), es específico de peculiaridades que responden al espacio por una parte regional (geográfico), y tiempo (época), históricamente determinado por la correlación de fuerzas, (ideologías), y la articulación de intereses de los distintos actores colectivos e individuales.

El resultado del proceso educativo en países de América Latina, en los que el régimen de organización por un largo tiempo fue la dictadura como el caso de Paraguay, con más de treinta y cinco años de dictadura, en la que la relación del Estado y la Ciudadanía fue de carácter vertical, con la tradición del partido único y fuerte, con la cultura de la obediencia, características esenciales de sistemas autoritarios.

Refleja una población que letarga en medio de una "comodidad" que representa la situación de la propia dictadura, todo queda a cargo del Estado. El punto de tensión radica en que la población del Paraguay no fue formada para el cambio de vida que significa la transición de 35 años de dictadura a la democracia y su exigencia (Fernández Bogado, 2013)

El cambio de paradigma exige, cambios en las políticas institucionales, en reconceptualizaciones. En la opinión de Arce (2019), la Educación Cívica, se consideró, durante muchos años, una asignatura más del currículo, sin resaltar el valor de la formación ciudadana y su impacto en la sociedad. Por tal motivo, considera que es necesario que las competencias ciudadanas se desarrollen en todas las asignaturas.

La investigación se justifica en la necesidad de contar con informaciones que aporten conocimiento sobre la necesidad de ajustar, conciliar enfoques teóricos con prácticas pedagógicas en el contexto democrático con el propósito de afianzamiento de competencias democráticas en los estudiantes.

El tema de la educación ciudadana en el ámbito educativo ha cobrado especial interés desde la última década del pasado siglo y, especialmente, en el comienzo del presente, tanto a nivel de cada país, como en contextos internacionales. Es obvio que ante los desafíos actuales que enfrentan países latino americanos en materia de cultura política y democratización de los espacios públicos y privados, emerge una tendencia de educación ciudadana que privilegia la formación para la acción transformadora desde una perspectiva crítica y contextualizada (Ríos Ojeda, RÍOS O. V. (2018). Educación Superior en el siglo XXI. V. Ríos , 2018). 
La literatura aborda la Educación en el contexto de la Democracia bajo la influencia del modelo económico que predominó en el Paraguay desde 1989, hasta nuestros días, desarrolla planteamientos enmarcados en la relación Educación, Democracia y Enfoques, ¿hasta qué punto la Educación posibilita la adquisición de habilidades democráticas? Incorpora en su análisis, experiencias de liderazgo estudiantil con énfasis en el nivel medio casos: Unión Nacional de Centros de Estudiantes del Paraguay - UNEPY., y Federación Nacional de Estudiantes Secundarios - FENAES. Espacios de participación estudiantil, democrático, pluralista, en la cual las opiniones, ideas propuestas y acciones son consensuales con el fin de encontrar soluciones a los problemas que afectan a la educación paraguaya.

El trabajo enfoca el estudio siguiendo la línea de Teóricos, que fundamentan en la Educación una formación con base en los valores éticos y democráticos como la manera de generar cambios significativos para la sociedad, entre los que se presentan, los aportes a la Educación de Ramón Indalecio Cardozo (1876-1943), en las primeras décadas del siglo XX, revolucionó la educación paraguaya con la introducción del escolanovismo. Las principales características de la Reforma 22/24 se pueden resumir en cuatro aspectos: el nacionalismo cívico, la educación laica, el pragmatismo y la educación y protección integral de niños y niñas. El nacionalismo cívico significaba, en el concepto del maestro, situar el cumplimiento de la ley como el primer deber a ser enseñado a los educandos. Esta idea de civismo estaba complementada con la idea del trabajo como medio para el "engrandecimiento de la patria", siguiendo, en este sentido a Kerschensteiner, para quien la comunidad de trabajo es el medio para el aprendizaje de los valores cívicos. Esta visión confiere al trabajo de un profundo sentido cívico-moral.

Educación democrática: entendida a lo largo de la investigación, como educación para la democracia, (superando la dicotomía del concepto liberal de Educación), El concepto de Dewey es un compromiso liberal que entiende el liberalismo, pero vinculado con el desarrollo del conocimiento y de la inteligencia (Pettit, 1999). Se plantea un enfoque en la que confluyan las teorías de una ciudadanía con elevada capacidad de participación en los asuntos públicos de la sociedad para mejorarla (Cortina, 1997). La consideración de la evolución de los niños, sus peculiaridades, y la búsqueda de los mejores modelos pedagógicos y psicológicos, propuestas resumidas en la escuela activa de Cardozo R. I., citado por (Velázquez Seiferheld, 2016). Una Educación para la gobernabilidad, los 
aportes en educación de Looke, y Rousseau, la Teoría del Desarrollo Humano la que concluye en que el ser humano es no solo objeto de la Educación; sino el medio y el fin.

Ciudadanía: la definición de ciudadanía a partir de la teoría educativa para la ciudadanía desde el enfoque de Cortina. El concepto de ciudadanía es fundamental en nuestra propuesta educativa, pues en él convergen los demás bloques: a) La ciudadanía significa, en primer lugar, igualdad ante la ley y la no discriminación por razones de sexo, etnia, creencias, etc. b) La ciudadanía significa libertad. En los últimos tiempos se ha propuesto un nuevo concepto de libertad, como un derecho y como un deber: participar e intervenir en la sociedad para mejorarla (Camps, 1993, Cortina, 1997). citado por Santisteban \& Pagés (2012), en La Educación Democrática de la ciudadanía: una propuesta conceptual, esta nueva idea de libertad ha surgido a partir de la necesidad de incluir en la educación, a)- la democracia entendida como participación. b)- la ciudadanía es justicia, supone unos derechos y unos deberes que dan sentido a nuestra participación en la sociedad y en la política. c)- la solidaridad alude al concepto de fraternidad. Sin solidaridad no existe la democracia, ya que es una organización social y un sistema político en el que existen desigualdades. Su perfeccionamiento o su construcción depende de nuestra capacidad para mejorar estas situaciones (Bilbeny, 2016).

Incorporando al análisis el tema Gobernabilidad y Educación, como señala G. O’Donnell (1996:87), citado por Filmus (2005), en Gobernabilidad democrática, conceptualización, en muchas de las sociedades latinoamericanas «[...] los individuos solo son ciudadanos en relación con la única institución que funciona en forma parecida a lo que prescriben sus reglas formales: las elecciones $[\ldots] »$. El fortalecimiento de la gobernabilidad requeriría, entonces, tanto del desarrollo de mecanismos alternativos de participación política de la población como de la profundización de una cultura política que permitiera ejercer una ciudadanía plena.

A fin de comprender posturas antagónicas, que se constituyeron en Los años 20 en Paraguay: nacionalismo en conflicto, "lopistas" y "antilopistas", o entre patriotas y "legionarios". Se recogen conceptualizaciones que reflejan la influencia de acontecimientos históricos-políticos desde Velázquez Seiferhel (2016), en su obra

\footnotetext{
${ }^{1}$ En el vocabulario político paraguayo la expresión "legionario" designa la actitud antinacional. El vocablo se refiere a la Legión Paraguaya, cuerpo militar creado por exiliados paraguayos en la Argentina para combatir a lado de las tropas aliadas durante la Guerra de la Triple Alianza (1865-1870). La evolución del término hizo que pasara a identificarse con la idea de traición a la patria o antinacionalismo.
} 
"Relaciones entre autoritarismo y Educación en el Paraguay 1869 - 2012”, el análisis que ofrece el autor cuando se analiza el período que va desde la Reforma Educativa de 1931 hasta 1954, el rasgo a destacar es el papel predominante del autoritarismo y el nacionalismo heroico en el discurso educativo paraguayo.

Al mismo tiempo Velázquez Seiferhel y Marielle Palau, socióloga, hacen referencia al: Protagonismo de Liderazgo Estudiantil. Paraguay: estudiantes forzaron la renuncia de la ministra de Educación (Báez, 2015).

Luego de tres días de protestas en todo el país, con toma de colegios, sentadas y marchas multitudinarias por tres días consecutivos, los estudiantes paraguayos precipitaron la renuncia de la ministra de Educación, Marta Lafuente.

\section{ESTRATEGIAS METODOLÓGICAS}

La investigación se desarrolló a través de la metodología cualitativa, tipo descriptivo, no experimental, transversal, se recolectaron datos en un solo momento. Su propósito fue describir variables y analizar su incidencia e interrelación en un momento dado, no habiendo manipulación intencional, ni asignación al azar. Los sujetos se clasificaron en un grupo o nivel determinado de la variable independiente por autoselección (Sampieri \& Mendoza Torres, 2010). Por medio de esta propuesta se posibilitó la abstracción del problema de la Educación en el contexto de la democracia actual, con análisis desde la interpretación bibliográfica, se conceptualizó, la función educativa paraguaya, se evaluó la adquisición de competencias, vinculadas con la experiencia de los Centros de Estudiantes de colegios del nivel medio, caso UNEPY y FENAES., se realizó la interacción de variables: política educativa - teorías - enfoques didácticos en la pedagogía para una formación en el contexto de la democracia.

Los métodos utilizados fueron: observación, mediante la cual se recolectan datos sobre variables, conceptualizadas en un contexto social, movilización, organización, y manifestación de lideres estudiantiles, ocurridas en Paraguay, estudiando al citado fenómeno, revisión bibliográfica, análisis de documentos, entrevistas dirigidas y encuestas. En el proceso de esta investigación, por la naturaleza del tema, complejo, y abarcante, se profundizó en la lectura de teóricos que analizaron la Democracia y la Educación, y se sintetizó sobre sus aportes, por medio de un enfoque cualitativo. 


\section{Población y Muestra}

Se ha recurrido a un muestreo probabilístico estratificado, la población se dividió en segmentos y se seleccionó una muestra para cada segmento o sea unidades de análisis: teóricos, profesionales en ejercicio de la educación en el nivel medio, terciario, y universitario, coordinadores académicos, directivos, docentes, alumnos de colegios en ejercicio del liderazgo estudiantil por medio de centros de estudiantes, casos UNEPY y FENAES.

\section{Validación de Instrumentos}

Dentro del marco de una investigación cualitativa, la triangulación, según Benavides \& Gómez Restrepo (2005), comprende el uso de varias estrategias al estudiar un mismo fenómeno, la triangulación ofrece la alternativa de poder visualizar un problema desde diferentes ángulos (sea cual sea el tipo de triangulación), y de esta manera aumentar la validez y consistencia de los hallazgos, la triangulación ofrece una oportunidad para que se elabore una perspectiva más amplia en cuanto a la interpretación del fenómeno en cuestión.

\section{RESULTADOS Y DISCUSIÓN}

Las entrevistas brindaron información sobre la variable presentada en interrogante: ¿cómo se enfoca la educación para la democracia? - Identificar teorías educativas.

- La política de educación está definida en la Ley Marco de Educación Ley N. ${ }^{\circ}$

- 1264/98, regula el nivel de la educación media, conceptualiza la educación como un bien público, en el marco la Declaración y Plan de Acción de la UNESCO., declaración Final de la Conferencia Mundial sobre la Educación y sus desafíos para el siglo XXI. La definición de bien público, implica: el nivel de formación, debe contribuir al desarrollo del país, bajo la condición de un modelo de gobierno, que es el democrático, garantizando la igualdad de condiciones de todos los ciudadanos para el acceso a la educación, y al desarrollo humano.

- La investigación arrojó como una de las debilidades del sistema educativo paraguayo, el alcance de la educación como bien público es relativo. Las brechas de desigualdades es un punto crucial que debe ser incluida en la agenda de la política educativa, objetivo a disminuir por medio de estrategias educativas

- Es primordial la reflexión del profesorado, acerca de cómo se está trabajando la 
- enseñanza de los transversales en función de la educación del componente educación democrática (Arévalos, 2018).

- De acuerdo a lo estipulado por el Currículum para Educación Media, no existe un método asignado, un procedimiento específico de cómo trabajar los ejes transversales. El término transversalidad, según Bisquerra (2012), citado por Rolín \& Balletbo Fernández (2018) "se refiere a unos contenidos que no están ligados a ninguna materia académica ordinaria".

- En cuanto a la educación universitaria Giménez Duarte (2018), sostiene entre otros aspectos, el principal documento de política pública de la educación universitaria en Paraguay, es denominado Libro Blanco, elaborado por el Consejo Superior Universitario, documento que debe fungir de marco orientador para los proyectos, programas y planes para la gestión de las instituciones, no sugiere ningún lineamiento, en forma clara y expresa, para la formación de profesionales, con base en los valores éticos y democráticos, puntualizó el autor al referirse a las políticas públicas en la educación universitaria desde la perspectiva ético-ciudadana.

Resultados de las encuestas y entrevistas a docentes, directores, coordinadores pedagógicos de la educación media, terciaria y universitaria.

Destacó el Predominio del Enfoque Capitalista en Educación, características que evidencian debilidades de la Educación, causadas por una marcada tendencia del enfoque neoliberal: Aún cuesta aplicar otro enfoque, no hay suficiente conocimiento sobre cómo proceder, no se cuenta con una guía sobre qué prácticas pedagógicas se ajustan exactamente para el afianzamiento de habilidades democráticas ético-ciudadana, desconocimiento del manejo adecuado de tecnologías, como herramientas pedagógicas, prevalece una cultura dictatorial.

Destacan la falta de espacios, en el currículum, exclusivos para el desarrollo de las habilidades democráticas, falta de enfoques integradores en la práctica. En educación falta partir de la realidad de contexto. Coincidiendo con Vera \& Velazquez Seiferheld (2019), quienes coinciden en ubicar el debate del problema de la educación paraguaya en las sugerencias de Cardozo, es el único que desarrolla con amplitud un sistema educativo, en base a realidades del contexto social.

A lo largo de la investigación se resalta una tendencia que evidencia la transversalidad de los principios democráticos en el currículum de la educación media, comporta un enfoque 
muy amplio con claros de especificidades para la apropiación de capacidades democráticas, se menciona la necesidad de incorporar el componente educación democrática en una disciplina específica dentro del currículo.

Como resultado de la encuesta aplicada a líderes estudiantiles, caso UNEPY., y FENAES., la mayoría, coincidieron en que no tienen alguna materia específica en el nivel medio, que aborde contenidos sobre: antecedentes, conceptos, características y alcance de los sistemas de gobiernos, mecanismos de participación en democracia, características y diferencias de las democracias en América Latina. Antecedentes y características de nuestra democracia, particularidades del Estado paraguayo en el contexto de la democracia actual, manejo oportuno y pertinente de una agenda política institucional. Aclararon que tienen conocimiento muy general sobre definiciones de Estado, política, religión, elecciones de autoridades, conceptos sobre Constitución Nacional, Congreso, y otros, sin embargo, se experimenta una sensación de falta de conexión entre lo que estudiamos y lo que realmente ocurre. Se estima como una debilidad latente en educación media, la ausencia de una materia específica destinada al desarrollo de actitudes democráticas en los educandos, para afianzar capacidades democráticas, y canalizar el liderazgo.

Predominó la postura de falta de acompañamiento, conceptualizada en orientación pertinente y oportuna en aspectos que hacen al ejercicio de liderazgo. Durante la entrevista, coincidieron en afirmar, que no se les forma en la capacidad ciudadana de pensamientos críticos, así mismo, recalcaron: "aún nos movemos en un ambiente en el que predomina el autoritarismo", la verticalidad de autoridades, incluso en nuestras propias instituciones educativas, colegios, y otros estamentos educativos.

Entre otros aspectos, recalcaron, hay veces que experimentamos sensación de orfandad con relación a nuestras autoridades del MEC., puesto que nos consideramos más que solo alumnos con obligaciones, el estudio en primer lugar, así también somos ciudadanos con derechos, queremos se nos tenga presente como actores claves de la Educación, se consideren, nuestras necesidades y aspiraciones al momento de proyectar y generar cambios en pos de una Educación de Calidad. "necesitamos mayor contacto entre nuestros pares; y formación" encarada por los propios coordinadores de la UNEPY, y FENAES. 


\section{CONCLUSIÓN}

Al conceptualizar, la función educativa paraguaya, en relación al contexto de las exigencias de la democracia, resalta la dicotomía, entre el estado social y el estado de derecho, con forma de gobierno la democracia con bases en principios del neoliberalismo. La crítica que la literatura política sostiene contra el estado de derecho, es que concentra su argumentación en un concepto de igualdad formal: todos somos iguales ante la ley, pero este modelo y concepción del estado no piensa en la desigualdad material existente entre los ciudadanos (Perona, 1993). En este marco de análisis se encontró que el alcance de la educación como bien público es relativo, brechas de desigualdades existen, desde la posibilidad de acceso a la educación.

La conceptualización del problema educación, democracia y capacidades democráticas, enmarcó la investigación en teorías fundamentadas, planteando como hipótesis que la cultura institucional, instalada por el enfoque capitalista en educación, ubicó como centro de atención de las políticas educativas, a las metodologías, desviándose de la esencia de la educación, el hombre, se evidenció el predominio del enfoque capitalista en educación, justificándose la hipótesis de investigación.

En relación al objetivo evaluar la adquisición de competencias, vinculadas con la experiencia de centros de estudiantes de colegios del nivel medio UNEPY y FENAES, se rescató como fortaleza la adquisición de competencias democráticas, vinculada a la experiencia de liderazgos. En cuanto al currículum del nivel medio ¿posibilita espacios de afianzamiento de capacidades democráticas? la segunda Hi.: El enfoque transversal de los principios democráticos en la educación paraguaya, no propicia aprendizaje significativo con relación a las exigencias de la democracia. En relación a esta postura, se considera como una debilidad latente en educación media, la ausencia de una materia específica destinada al desarrollo de actitudes democráticas, para afianzar capacidades democráticas, y canalizar el liderazgo.

\section{LISTA DE REFERENCIAS}

Arce, H. (2019). Competencias ciudadanas: una reconstrucción conceptual en el marco de la Educación Cívica costarricense. Revista Actualidades Investigativas en Educación, 19(2), 1-20. .

Arévalos, L. (2018). I Congreso de Educación y Psicopedagogía. Encarnación. 
Báez, C. (02 de Octubre de 2015). PARAGUAY.com. Obtenido de PARAGUAY.com: https://paraguay.com/

Benavides, M. O., \& Gómez Restrepo, C. (2005). Métodos en investigación cualitativa: triangulación. Revista Colombiana de Psiquiatría, 118-124.

Bilbeny, N. (2016). Justicia Compasiva. La justicia como cuidado de la existencia. Tecnos.

Bisquerra, R. (2018). Educación emocional para el desarrollo integral en secundaria. Obtenido de http://www.ub.edu/grop/wpcontent/uploads/2018/11/Educaci\%C3\%B3n-emocional-para-el-desarrollointegral-en-secundaria.pdf

Cortina, A. (1997). Ciudadanos del mundo: Hacia una teoría de la ciudadanía. Madrid: Alianza Editorial.

Estébanez, P. F. (1997). Teoría de la Educación. Madrid : Ediciones Agulló.

Fernández Bogado, B. (2013). Clase magistral: Desafíos de la Educación paraguaya en el siglo XXI curso de posgrado doctorado en Ciencias de la Educación con énfasis en investigación científica. Facultad de Humanidades y Ciencias de la Educ., UNP. . Pilar.

Filmus, D. (2005). Gobernabilidad democrática: conceptualización. Revista electrónica Aportes Andinos;No. 13.

Giménez Duarte, F. J. (2018). Formación ético-ciudadana. Novapolis.

Perona, Á. (1993). El Liberalismo y la Socialdemocracia: Popper y la sociedad abierta. Barcelona: Anthropos.

Pettit, P. (1999). Republicanismo. Paidos.

Ríos Ojeda, V. (julio de 2018). RÍOS O. V. (2018). Educación Superior en el siglo XXI. V. Ríos . Pilar.

Ríos Ojeda, V. (Julio de 2018). Conferencia Magistral llevada a cabo en el marco de la semana de actos evocativos por la Fundación de la Universidad Nacional de Pilar a cargo de la Facultad de Humanidades y Ciencias de la Educación UNP. Paraguay. Educación Superior en el siglo XXI. Pilar.

Rolín, E. D., \& Balletbo Fernández, I. (2018). La educación en valores en el sistema educativo paraguayo. Obtenido de DocPlayer: http://docplayer.es/185336372- 
Titulo-la-educacion-en-valores-en-el-sistema-educativo-paraguayo-education-invalues-in-the-paraguayan-educational-system.html

Sampieri, R. H., \& Mendoza Torres, C. (2010). Metodología de la investigación. Mc Graw Hill Education.

Santisteban, A., \& Pagés, J. (26 de Junio de 2012). CORE. Obtenido de CORE: core.ac.uk/download/pdf/41584104.pdf

Velázquez Seiferheld, D. (Diciempre de 2016). Relaciones entre autoritarismo y educación en el Paraguay 1869 - 2012. Un Análisis Histórico. . Pilar, Paraguay: SERPAJ PY.

Velázquez Seiferheld, D. (viernes 17 de diciembre de 2018). Política Educativa en el Paraguay. (L. V. Cuevas, Entrevistador)

Vera, A. S., \& Velazquez Seiferheld, D. (2019). Ramón Indalecio Cardozo (1876-1943). Pionero de la Escuela Activa paraguaya. Revista historica de la educación latinoamerica, 15-34. 